\title{
RESEARCH HIGHLIGHT A spatial map out of place
}

\author{
Adrien Peyrache $\mathbb{D}^{1}$ and Adrian J. Duszkiewicz ${ }^{1,2}$ \\ Cell Research (2021) 31:605-606; https://doi.org/10.1038/s41422-021-00478-w
}

\begin{abstract}
The ability of mammals to navigate large and ever-changing environments requires sophisticated computations and representations that are usually thought to depend on the hippocampus and associated structures. In a recent study published in Cell Research, Long et al. report that neurons of the primary somatosensory cortex, recorded in freely moving rats, show various spatial correlates reminiscent of those of the hippocampal-entorhinal system, raising new questions about the prevalence of spatial coding in the mammalian brain.
\end{abstract}

The discovery of hippocampal place cells ${ }^{1}$-neurons that each fires at a specific location of the animal in its environment-has laid the foundation for the idea that the brain contains cognitive spatial maps. ${ }^{1,2}$ The central role of the hippocampus in spatial navigation was further supported by lesion studies. ${ }^{3}$ The discoveries of the brain's head direction system ${ }^{4}$ and other complex spatial representations in parahippocampal structures such as grid cells that fire at the vertices of a hexagonal grid regularly tessellating the environment, ${ }^{5}$ provided further evidence for the role of the hippocampal-entorhinal system in map-based navigation.

The exclusive role of the hippocampus in spatial mapping has been undermined by a series of reports showing spatially-tuned neurons in various other cortical areas. Notably, the visual cortex in mice encodes position with the same accuracy as the hippocampus, ${ }^{6}$ and a recent report confirmed distributed nature of spatial coding across most, if not all, regions of the dorsal cortex, ${ }^{7}$ including somatosensory and motor cortices. Importantly, the emergence of these representations requires an intact hippocampus. ${ }^{7}$ Additionally, most studies reporting spatial coding outside of the hippocampus have a major caveat: they were performed in animals exploring linear tracks, either freely moving or head fixed and running on treadmills, hindering the possibility of examining more complex spatial neural correlates. One possibility is that the spatial tuning described in these findings corresponds only to a low-level computation of one direction of movement that may indirectly depend on hippocampal processing without complex links.

Using standard recording techniques in freely moving rats exploring an open field, Long et al. ${ }^{8}$ examined the spatial correlates of neurons in deep layers of the primary somatosensory cortex. Unexpectedly, they found that $\sim 10 \%$ of recorded neurons showed spatially firing patterns reminiscent of the place cells in the hippocampus. Importantly, these spatial firing fields displayed many properties that define hippocampal place fields: they uniformly covered the whole environment, were stable across the recording session, and rotated in concert with a distal visual cue and remapped in a new environment.

While other spatially modulated cell types are scarce in the hippocampus and are instead enriched in parahippocampal structures like entorhinal cortex and the subicular complex, Long et al. ${ }^{8}$ found that in deep layers of primary somatosensory cortex place cells were present alongside a whole repertoire of spatially tuned neuron types: grid cells, border cells and head direction cells. While head direction and border tuning were relatively common ( $17 \%$ and $12 \%$ of recorded cells, respectively), grid-like tuning was scarcer, constituting $<4 \%$ of all recorded neurons. All of these spatially modulated cell types behaved like their entorhinal cortex equivalents in response to environmental manipulations: head direction cells followed a rotated visual cue, border cells showed a new border field in response to a novel environmental border and grid cells maintained their hexagonal firing patterns in a new, larger enclosure. Importantly, this spatial tuning could not be explained by any other variables: the presence of place field was not dependent on the animal's running speed and the directional tuning of head direction cells did not depend on the angular velocity of head movements. Interestingly though, spatial tuning of place, grid, and border cells was less defined during immobility, possibly mirroring non-local activity of hippocampal place cells during similar behavioural states. Spatial tuning was also not affected by trimming of the rat's whiskers, indicating that while this sensory modality is a major input to the rodent primary sensory cortex, it does not seem to influence the local spatial map. Together, these landmark findings constitute the first discovery of a complete spatial mapping system outside of the hippocampal-entorhinal circuit.

The key question is now to determine whether this spatial map is computed locally or is instead relayed from the hippocampus and/or the parahippocampal regions. The medial entorhinal cortex shares mutual connections with many cortical regions and such spatial codes can be inherited from feedback signals that enable reliable integration of multimodal sensory streams necessary for navigation. Could these spatial features emerge from local computation? A response does not necessarily require inactivation or lesions of the parahippocampal regions. Careful environmental manipulations and further analysis of the data will provide further evidence towards locally generated versus inherited code. For example, hippocampal place cells acquire a place field at the very first visit by the animal, and establishing whether this property is shared by cortical location-tuned neurons will broaden our understanding of the exact nature of this code. Another key property of a population of place cells is replaying encoded trajectories during immobility and sleep. ${ }^{9}$ It would be interesting to see whether spatially tuned neurons in the primary somatosensory cortex engage in similar experience-dependent replay and whether such non-local activity is coordinated with or independent of the hippocampus.

What does it mean for the somatosensory cortex to possess such a spatial code? The somatosensory cortex is obviously not a

${ }^{1}$ Montreal Neurological Institute, McGill University, Montreal, QC H3A 2B4, Canada and ${ }^{2}$ Centre for Discovery Brain Sciences, The University of Edinburgh, Edinburgh, UK

Correspondence: Adrien Peyrache (adrien.peyrache@mcgill.ca)

Published online: 24 February 2021 
simple relay of sensory inputs to downstream higher-order cortical areas that determine whether to act upon incoming messages. In contrast, the somatosensory cortex can be conceptualised as an active model of the animal in motion, constantly dependent on both present contexts and possible future actions. ${ }^{10}$ Hence, as the animal explores its environment, the representation of current and future states by the somatosensory circuits could incorporate a spatial mapping component.

In conclusion, the study by Long et al. opens new avenues for our understanding of the neuronal basis of cognition and will undoubtedly lead to new studies examining the origin and nature of this spatial code. Ultimately, one can wonder: Is everything in the mind really about space? Let us finish by reminding what Kant had proposed in his Inaugural Dissertation, long before the first action potential was recorded: "[space] is subjective and ideal, and originates from the mind's nature in accord with a stable law as a scheme, as it were, for coordinating everything sensed externally".

\section{ADDITIONAL INFORMATION}

Competing interests: The authors declare no competing interests.

\section{REFERENCES}

1. O'Keefe, J. \& Nadel, L. Oxford Cognitive Maps in Rats and Men (Oxford University Press, 1978)

2. Tolman, E. C. Psychol. Rev. 55, 189-208 (1948).

3. Morris, R. G. M., Garrud, P., Rawlins, J. N. P. \& O'Keefe, J. Nature 297, 681-683 (1982).

4. Taube, J. S., Muller, R. U. \& Ranck, J. B. J. Neurosci. 10, 420-435 (1990).

5. Hafting, T., Fyhn, M., Molden, S., Moser, M. B. \& Moser, E. I. Nature 436, 801-806 (2005).

6. Saleem, A. B., Diamanti, E. M., Fournier, J., Harris, K. D. \& Carandini, M. Nature 562, 124-127 (2018).

7. Esteves, I. M. et al. J. Neurosci. 41, 307-319 (2021).

8. Long, X. \& Zhang, S. J. Cell Res. https://doi.org/10.1038/s41422-020-00448-8 (2021).

9. Lee, A. K. \& Wilson, M. A. Neuron 36, 1183-1194 (2002).

10. Brecht, M. Neuron 94, 985-992 (2017). 